\title{
ON THE SET OF EXTREME POINTS OF A CONVEX BODY
}

\author{
JAMES B. COLLIER
}

ABSTRACT. We prove the following: Given a subset $X$ of a compact 0 -dimensional metric space $Z$ and an integer $d \geq 3$, there is a homeomorphism of $Z$ into the boundary of a convex body $C$ in $E^{d}$ mapping $X$ onto the set of extreme points of $C$ if and only if $X$ is a $G_{\delta}$ set with at least $d+1$ points.

A convex body is any closed bounded convex set with nonempty interior. It is well known that the set of extreme points of a convex body in $E^{d}$ is a $G_{\delta}$ set with at least $d+1$ points. We may consider as a partial converse to this the fact that for each integer $n \geq d+1$ there is a convex body in $E^{d}$ with exactly $n$ extreme points. The purpose of this note is to prove a more general converse. We denote the set of extreme points of a convex set $C$ by ext $C$. Our main result is

Theorem 1. Let $X$ be a subset of a compact 0-dimensional metric space $Z$, and let $d$ be an integer with $d \geq 3$. There is a homeomorphism of $Z$ in. to the boundary of a convex body $C$ in $E^{d}$ mapping $X$ onto ext $C$ if and only if $X$ is a $G_{\delta}$ set with at least $d+1$ points.

The boundary of any convex body in $E^{d}$ is homeomorphic to the $(d-1)$ sphere, $S^{d-1}$. For each positive integer $d$ we define $\chi_{d}$ to be the family of all subsets $X$ of $s^{d-1}$ for which there is a homeomorphism of $s^{d-1}$ onto the boundary of a convex body $C$ in $E^{d}$ mapping $X$ onto ext $C$. The family we call $\chi_{3}$ was first defined in [3] and the question raised of finding a characterization of that family. It is known that each member of $\chi_{d}$ is a $G_{\delta}$ set with at least $d+1$ points and that each closed subset of $s^{d-1}$ with at least $d+1$ points is a member of $\chi_{d}$. However, no proper open subset of $S^{d-1}$ is in $\chi_{d}$. Using Theorem 1 we may further state

Corollary 1. Any $G_{\delta}$ subset of $S^{2}$ with at least four points and whose closure is 0-dimensional is a member of $\chi_{3}$.

Received by the editors October 30, 1973 and, in revised form, December 17, 1973.

AMS (MOS) subject classifications (1970). Primary 52A15, 52A20. 
Corollary 2. For $d \geq 3$ any subset of $S^{d-1}$ with at least $d+1$ points and whose closure is countable is a member of $\chi_{d}$.

These follow easily from results in $[4$, p. 532] and $[2$, p. 456], respectively, on extending homeomorphisms.

We proceed to the proof of Theorem 1. The closure, the boundary, and the convex hull of a subset $A$ of $E^{d}$ will be denoted by $\mathrm{cl} A$, bd $A$, and conv $A$, respectively. For any compact subset $K$ of the real line $\mathbf{R}, \mathbf{R} \backslash K$ is the disjoint union of countably many open intervals. Let $e(K)$ be the set of endpoints of these intervals; then $e(K)$ is a countable subset of $K$.

Lemma 1. Let $Y$ be a compact 0 -dimensional subset of $\mathbf{R}$, and $X$ a $G_{\delta}$ subset of $Y$ which contains $e(Y)$. Then there is a continuous function $g: Y \rightarrow \mathbf{R}$ such that $X \cap g^{-1}(s)$ is the set of extreme points of conv $g^{-1}(s)$ for each $s \in g[Y]$.

Proof. We construct a sequence $\mathcal{U}_{0}, \mathcal{U}_{1}, \ldots$ of open (and also closed) coverings of $Y$ by pairwise disjoint sets so that for each $n \geq 0, \mathcal{U}_{n+1}$ is a refinement of $\mathcal{U}_{n}$. For each $U$ in $\mathcal{U}_{n}$ we shall specify a compact subset $c_{n}(U)$ of $U$.

Since $X$ is a $G_{\delta}$ set, $Y \backslash X$ is the countable union of a nested chain $K_{1} \subseteq K_{2} \subseteq$. . of compact sets. We may choose $\mathcal{U}_{0}=\{Y\}$ and $c_{0}(Y)=\varnothing$ and suppose that $\mathcal{U}_{n-1}$ and $c_{n-1}$ have been defined for some $n \geq 1$. To complete the inductive construction, we need only specify for an arbitrary $U \in \mathcal{U}_{n-1}$ the members $V_{0}, \ldots, V_{k}$ of $\mathcal{U}_{n}$ which are subsets of $U$ and define $c_{n}\left(v_{i}\right)$ for $i=0, \cdots, k$. Since $U$ is compact and 0 -dimensional, there is a finite open covering $\mathcal{O}$ of $U$ by pairwise disjoint sets each of diameter $\leq 1 / n$. Let $V_{0}$ be the union of those members of $\mathcal{C}$ which intersect $c_{n-1}(U)$ and let $V_{1}, \ldots, V_{k}$ be an enumeration of the remaining sets in $\mathcal{O}$. Choose $c_{n}\left(V_{0}\right)=c_{n-1}(U)$. By hypothesis the extreme points $a_{i}, b_{i}$ of conv $V_{i}$ are in $X$. We define $c_{n}\left(V_{i}\right)=\left\{a_{i}, b_{i}\right\} \cup\left(K_{n} \cap V_{i}\right)$ for $i=1, \ldots, k$. Hence the extreme points of conv $c_{n}\left(V_{i}\right)$ are $a_{i}, b_{i}$ and these are the only points of $c_{n}\left(V_{i}\right)$ which lie in $X$.

Consider each $\mathcal{U}_{n}$ to be a topological space with the discrete topology. Then there is a continuous mapping $f_{n}: \mathcal{U}_{n} \rightarrow \mathcal{U}_{n-1}, n \geq 1$, defined by ' $\subseteq f_{n}(U)$, and there is a continuous mapping $g_{n}: Y \rightarrow \mathcal{U}_{n}$ defined by $y \in$ $g_{n}(y)$. Thus $\left\{\mathcal{U}_{n}, f_{n}\right\}$ is an inverse limit sequence. Let $\mathcal{U}_{\infty}$ be the inverse limit space of $\left\{\mathcal{U}_{n}, f_{n}\right\}$, and let $g_{\infty}: Y \rightarrow \mathcal{U}_{\infty}$ be the continuous mapping induced by $g_{n}: Y \rightarrow \mathcal{U}_{n}$. Since $Y$ is compact and $\mathcal{U}_{n}$ is discrete for each $n \geq$ $0, \mathrm{U}_{\infty}$ is compact and 0 -dimensional; hence there is a homeomorphism 
$h: \mathcal{U}_{\infty} \rightarrow \mathbf{R}$. We define $g=h \circ g_{\infty}$. From the construction of $g_{\infty}$ it is evident that for $x, y \in Y, g(x)=g(y)$ if and only if either $x=y$ or $x, y \in c_{n}(U)$ for some $U \in \mathcal{U}_{n}$ and $n \geq 0$. Since each point in $Y \backslash X$ lies in some $c_{n}(U)$ but is not an extreme point of conv $c_{n}(U), g$ has the desired property.

The next lemma may also be proved using inverse limit spaces. However, we omit the details since the method is similar to that used in $\left[1, \S \S_{2}-\right.$ $15]$.

Lemma 2. Let $Y$ be a compact, 0-dimensional metric space and $X$ a countable dense subset of $Y$. Then there is a homeomorphism $h: Y \rightarrow \mathbf{R}$ such that $e(h[Y])=h[X]$.

Proof of Theorem 1. One direction is immediate. To prove the other direction, suppose $X$ is an infinite $G_{\delta}$ subset of $Z$. Lemma 2 implies that we may assume $\operatorname{cl} X=Y \subseteq \mathbf{R}$ with $e(Y) \subseteq X$. Let $g: Y \rightarrow \mathbf{R}$ be as in Lemma 1 , and define $G: Y \rightarrow E^{3}$ by $x \rightarrow\left(x, g(x), g^{2}(x)\right)$. This is a homeomorphism of $Y$ into the graph of the convex function on $E^{2}$ given by $(x, y) \rightarrow y^{2}$. Let $C=\operatorname{conv} G[Y]$, then ext $C=G[X]$. Since we may extend $G$ to a homeomorphism $h: Z \rightarrow$ bd $C$, we have established Theorem 1 for $d=3$.

Assume the theorem is true for $d=n$, and express $Z$ as the disjoint union of closed subsets $Z_{1}, Z_{2}$ so that $X_{i}=X \cap Z_{i}$ is infinite, $i=1,2$. By assumption there is a convex body $C_{i}$ in $E^{n}$ and a homeomorphism $h_{i}: Z_{i} \rightarrow$ bd $C_{i}$ with $h_{i}\left[X_{i}\right]=$ ext $C_{i}$. We may consider $C_{1}, C_{2}$ to lie in distinct parallel hyperplanes in $E^{n+1}$. Let $C=\operatorname{conv}\left(C_{1} \cup C_{2}\right)$ and define the homeomorphism $h: Z \rightarrow$ bd $C$ by $h / Z_{i}=h_{i}, i=1,2$. Then $C$ is a convex body in $E^{n+1}$ and $h[X]=\operatorname{ext} C$.

\section{REFERENCES}

1. J. G. Hocking and G. S. Young, Topology, Addison-Wesley, Reading, Mass., 1961. MR 23 \# A2857.

2. V. L. Klee, Homogeneity of infinite-dimensional parallelotopes, Ann. of Math. (2) 66 (1957), 454-460. MR 20 \# 1200.

3. - Extremal structure of convex sets. II, Math. Z. 69 (1958), 90-104. MR 19, 1065.

4. K. Kuratowski, Topology. Vol. II, 3rd ed., Monografie Mat., Tom 21, PWN, Warsaw, 1961; English transl., PWN, Warsaw, 1968. MR 24 \# A2958; 41 \#4467.

DEPARTMENT OF MATHEMATICS, UNIVERSITY OF SOUTHERN CALIFORNIA, LOS ANGELES, CALIFORNIA 90007 